\title{
Determinação do teor de Ibuprofeno em Medicamento de Referência, Genérico e Similar, por Espectrofotometria Ultravioleta-Visível, em uma Farmácia Comunitária do Município de Jaguaquara, Bahia
}

\author{
Odiney dos Santos Souzal ; Caio Matheus da Rocha Coqueiro Monteiro Oliveira ${ }^{2}$
}

\begin{abstract}
Resumo: O Ibuprofeno é um medicamento inserido na classe dos anti-inflamatórios não esteroidais (AINES) e possui um extenso uso terapêutico principalmente no alívio da dor aguda e estados febris. Ao passo que é um medicamento comumente utilizado por diversas faixas etárias da população, faz-se necessário averiguar as condições de produção desse medicamento nos laboratórios. Através de uma rigorosa metodologia baseada na Farmacopéia Brasileira, pode-se avaliar o teor de concentração de princípios ativos presentes. Este presente trabalho teve como objetivo analisar o teor de comprimidos de ibuprofeno na dosagem de $600,0 \mathrm{mg}$ dentre marcas de genéricos, referência e similares comercializadas em uma farmácia comunitária privada do município de Jaguaquara, Bahia. Após a análise através da espectrofotometria obteve-se os seguintes resultados: As amostras de medicamentos de referência apresentaram massas de R1 = 429,23 mg; R2 = 367,69 mg; R3 = 429,23 e a média teor de concentração igual 68,11\%. Os medicamentos genéricos apresentaram valores de massa iguais a $\mathrm{G} 1=583,07 \mathrm{mg} ; \mathrm{G} 2=398,46 \mathrm{mg} ; \mathrm{G} 3=721,53 \mathrm{mg}$ e a média teor de concentração igual a 94,61\%. Os medicamentos similares apresentaram massas de S1 = 675,38 mg; S2 506,153 mg; S3= $660,0 \mathrm{mg}$ e a média teor de concentração de 102,30\%. Após os resultados das análises, observou-se que apenas as amostras dos comprimidos de genéricos e similares atingiram a média de teor de concentração preconizada pela Farmacopéia Brasileira, acusando uma possível falha nas Boas Práticas de Fabricação e Controle de Qualidade.
\end{abstract}

Palavras-chave: ibuprofeno; controle de qualidade; análise de teor.

\section{Determination of the Ibuprofen content in Reference, Generic and Similar, by Ultraviolet-Visible Spectrophotometry, at a Community Pharmacy in the Municipality of Jaguaquara, Bahia}

\begin{abstract}
Ibuprofen is a drug in the class of non-steroidal anti-inflammatory drugs (NSAIDs) and is one of the primary therapeutic treatments primarily for acute pain and febrile conditions. The process that is commonly used for the different age groups of the population, it is necessary to check the conditions of production of the drug in laboratories. The objective of this study was to obtain the theorem of ibuprofen tablets in the dosage of $600.0 \mathrm{mg}$ of generic brands, reference and commercialization in a private pharmacy by means of a rigorous methodology based on the Brazilian Pharmacopoeia, the municipality of Jaguaquara, Bahia. After an analysis by means of advanced spectrophotometry the results results: As reference mass images of $\mathrm{R} 1=429.23 \mathrm{mg} ; \mathrm{R} 2=367.69 \mathrm{mg} ; \mathrm{R} 3=429.23$ and a session content of $68.11 \%$. Generic drugs are equal to $\mathrm{G} 1=583.07 \mathrm{mg} ; \mathrm{G} 2=398.46 \mathrm{mg} ; \mathrm{G} 3=721.53 \mathrm{mg}$ and a movement index equal to 94.61\%. Similar drugs are masses of S1 $=675.38 \mathrm{mg} ; \mathrm{S} 2506.153 \mathrm{mg} ; \mathrm{S} 3=660.0 \mathrm{mg}$ and a mean concentration of $102.30 \%$. After the results of the analyzes, it was observed that the generic and similar tablets reached a mean concentration level recommended by the Brazilian Pharmacopoeia, and there was also a failure in Good Manufacturing and Quality Control Practices.
\end{abstract}

Keywords: ibuprofen; quality control; content analysis.

\footnotetext{
${ }^{1}$ Faculdade Independente do Nordeste. Cotato: neyjagua@ hotmail.com;

${ }^{2}$ Graduação em Ciências Farmacêuticas pela Universidade Estadual de Feira de Santana. Mestrado em Química pela Universidade Estadual do Sudoeste da Bahia. Professor da Faculdade Independente do Nordeste.
} 


\section{Introdução}

Os anti-inflamatórios não esteroidais (AINEs) estão entre as classes de medicamentos mais utilizadas pela sociedade e apresentam características antiinflamatórias, analgésicas e antitérmicas, sendo úteis para o controle da dor aguda leve e diminuição da febre (FIGUEIREDO; ALVES, 2015).

Dentre os diversos medicamentos da classe dos AINEs, o Ibuprofeno é um fármaco extensamente utilizado por diversos pacientes das mais variadas faixas etárias apresentam várias formas farmacêuticas e possui menos reações adversas em relação aos demais antiinflamatórios não esteroidais (GONDIM et al., 2017). O ibuprofeno age inibindo a enzima ciclooxigenase, inibindo a síntese de prostaglandinas, substâncias relacionadas com a inflamação (OLIVEIRA; CAMPOS, 2014).

Para garantir o padrão de qualidade de fabricação do ibuprofeno, o mesmo passa por alguns testes de qualidade que possam comprovar e satisfazer os princípios de pureza, eficácia, atividade e inocuidade (SILVA et al., 2014). Um dos testes empregados na determinação da porcentagem do teor de ibuprofeno em comprimidos é a técnica de espectrofotometria na região do ultravioleta de acordo com a metodologia descrita na Farmacopéia Brasileira (ANVISA, 2010). A Farmacopéia determina um espectro de absorção na faixa de 240 a 300 nanômetros, da solução a $0,025 \%$ (p/v) em hidróxido de Sódio 0,1 $M$ (ANVISA, 2010).

Ao realizar testes para assegurar a qualidade e o teor de um determinado medicamento é importante para garantir que o tratamento do paciente que adquire o medicamento seja de fato eficaz e que traga melhorias para sua condição de saúde (ROVERI et al., 2012).

O objetivo deste trabalho é verificar o teor de qualidade do ibuprofeno comercializado em uma farmácia comunitária privada do município de Jaguaquara, Bahia, a partir de um padrão de referência escolhido com a finalidade de proporcionar resultados satisfatórios quanto à qualidade do medicamento em questão. 


\section{Metodologia}

A metodologia do trabalho é do tipo experimental no qual utilizou-se para determinação do teor de ibuprofeno, o método proposto por Storpirtis et al (1999) que emprega a análise espectrofotométrica na região ultravioleta (UV) em meio alcalino. Foi usado o espectrofotômetro (QUIMIS U2M) selecionando o comprimento de onda 264 nanômetros.

\section{Amostras}

Os comprimidos revestidos de Ibuprofeno contendo $600 \mathrm{mg}$ foram adquiridos em uma farmácia comunitária privada do município de Jaguaquara, Bahia, provenientes de laboratórios renomados e conhecidos, designados como referência $(R)$, genérico $(G)$ e similar (S), no qual contém lotes diferentes de amostra comercial.

\section{Padrão de Referência (Ibuprofeno)}

O padrão analítico de referência do Ibuprofeno foi cedido gentilmente por uma Farmácia de Manipulação localizada no município de Jequié-BA, com alto grau de pureza conforme certificado de análise (ANEXO 01).

\section{Reagentes e solventes}

O hidróxido de sódio $(\mathrm{NaOH})$ 0,1 M foi empregado como solvente no preparo das soluções das amostras e do padrão. Todos os reagentes e solventes manuseados foram de alto grau de pureza.

\section{Procedimento}

A solução estoque foi preparada pesando-se $0,1000 \mathrm{~g}$ do padrão de ibuprofeno em um béquer dissolvendo-se com $60 \mathrm{~mL}$ de $\mathrm{NaOH} 0,1 \mathrm{~mol} / \mathrm{L}$ e transferido para um balão 
volumétrico de $100 \mathrm{~mL}$ acrescentando com água destilada obtendo uma solução de 1000 $\mathrm{mg} / \mathrm{L}$ (solução estoque).

A curva de calibração foi construída utilizando-se os padrões com as seguintes concentrações: 1, 2, 4, 8, 10, 16, 20, 30, 40, $50 \mathrm{mg} / \mathrm{L}$. No preparo dos padrões pipetou-se 0,1 $\mathrm{mL}(1 \mathrm{mg} / \mathrm{L}), 0,2 \mathrm{~mL}(2 \mathrm{mg} / \mathrm{L}), 0,4 \mathrm{~mL}(4 \mathrm{mg} / \mathrm{L}), 0,8 \mathrm{~mL}(8 \mathrm{mg} / \mathrm{L}), 1,0 \mathrm{~mL}(10 \mathrm{mg} / \mathrm{L})$, 1,6 mL (16mg/L), 2,0 mL (20mg/lL), 3,0 mL (30mg/L), $4 \mathrm{~mL}(40 \mathrm{mg} / \mathrm{L})$ e 5,0mL $(50 \mathrm{mg} / \mathrm{L})$ usando pipeta volumétrica e tendo o cuidado de completar o volume do balão volumétrico de 100,00 ml com $60 \mathrm{~mL}$ de $\mathrm{NaOH}$ 0,1 mol/L e o restante com água destilada usando pipeta de Pasteur, verificando o menisco.

Foram adquiridas as amostras em farmácia de dispensação da cidade de JaguaquaraBa sendo denominadas de medicamentos Referência (lote :LB17C1742/, validade - 03/19, Genérico (lote: 18B20I / validade - 02/20 e similar (lote: 412194/, validade - 03/20).

Cada comprimido revestido da amostra de ibuprofeno dita como referência, genérico e similar foi aberta num gral e pistilo, triturado em triplicata e dissolvido em $60 \mathrm{~mL}$ de $\mathrm{NaOH}$ 0,1 mol/L e depois transferiu-se todo o conteúdo para um balão volumétrico de $100 \mathrm{~mL}$ posteriormente acrescentado água destilada até o menisco. Em seguida, procedeu a diluição e dessas fez a leitura da absorbância no espectrofotômetro.

\section{Resultados e Discussão}

A partir do que pode ser observado na figura 01, a curva de calibração do ibuprofeno apresenta uma boa faixa de linearidade, reproduzindo valores de coeficiente de regressão linear $\mathrm{R}^{2}=0,9974$, que está de acordo com o valor preconizado pela Resolução $\mathrm{n}^{\circ}$ 899/2003, de coeficiente de correlação igual a 0,99. 
Figura 01. Curva de calibração do Ibuprofeno.

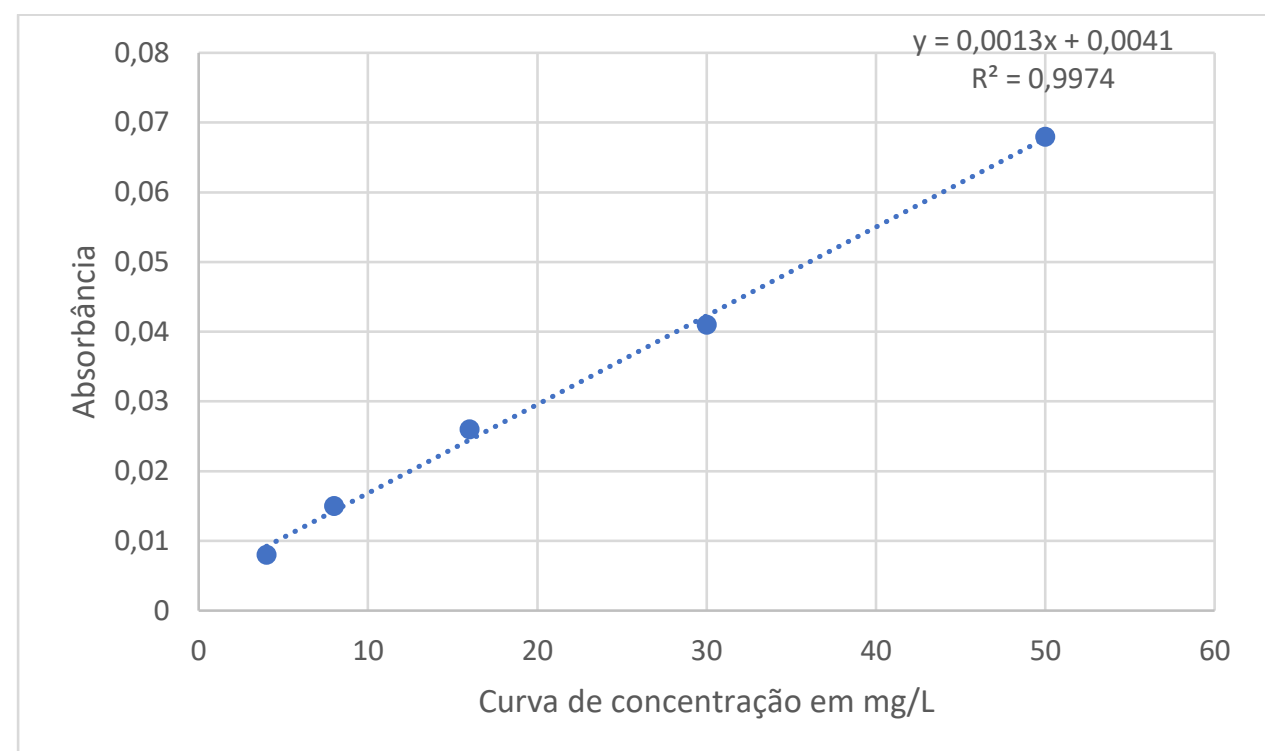

Fonte: Dados obtidos na pesquisa.

Foram utilizadas 01 amostra de medicamento denominado referência com análises em triplicata (R1,R2,R3), 01 amostra de medicamento denominado genérico com análises em triplicata $(\mathrm{G} 1, \mathrm{G} 2, \mathrm{G} 3)$ e 01 amostra de medicamento similar com amostras em triplicata (S1,S2,S3). Após a análise através da espectrofotometria obteve-se os seguintes resultados: As amostras de medicamentos de referência apresentaram massas de R1 = 429,23 mg; R2 = 367,69 mg; R3 = 429,23 e a média teor de concentração igual 68,11\%. Os medicamentos genéricos apresentaram valores de massa iguais a $\mathrm{G} 1=583,07 \mathrm{mg} ; \mathrm{G} 2=398,46 \mathrm{mg}$; G3= $721,53 \mathrm{mg}$ e a média teor de concentração igual a 94,61\%. Os medicamentos similares apresentaram massas de $\mathrm{S} 1=675,38 \mathrm{mg} ; \mathrm{S} 2$ 506,153 mg; $\mathrm{S} 3=660,0 \mathrm{mg}$ e a média teor de concentração de $102,30 \%$.

Segundo a Farmacopeia Brasileira (2010) a quantidade ideal de concentração de ibuprofeno que deve ser encontrada em um comprimido deve compreender em uma faixa de $90,0 \%$ a $110,0 \%$ de média de teor de concentração. A partir desse dado coletado da Farmacopéia, pode se observar que as amostras do medicamento de referência não se enquadram no preconizado em termos de teor de concentração de princípio ativo, sendo as amostras de medicamentos similares e genéricos encontrados dentro da faixa de aceitação de teor de concentração (TABELA 01). 
Tabela 01. Resultado da análise das amostras de Ibuprofeno.

\begin{tabular}{ccccc}
\hline Amostra & $\begin{array}{c}\text { Massa de Ibuprofeno } \\
(\mathrm{mg})\end{array}$ & Teor $(\%)$ & $\begin{array}{c}\text { Intervalo de } \\
\text { aceitação }\end{array}$ & Resultado \\
\hline R1 & 429,23 & 71,53 & $90-110$ & Reprovado \\
R2 & 367,69 & 61,28 & $90-110$ & Reprovado \\
R3 & 429,23 & 71,53 & $90-110$ & Reprovado \\
G1 & 583,07 & 97,17 & $90-110$ & Aprovado \\
G2 & 398,46 & 66,41 & $90-110$ & Reprovado \\
G3 & 721,53 & 120,25 & $90-110$ & Reprovado \\
S1 & 675,38 & 112,56 & $90-110$ & Reprovado \\
S2 & 506,15 & 84,35 & $90-110$ & Reprovado \\
S3 & 660,0 & 110 & $90-110$ & Aprovado \\
\hline
\end{tabular}

Fonte: Dados da pesquisa.

O uso de medicamentos com concentrações de princípio ativos inferiores ou superiores a que foi declarada em seu rótulo pode trazer uma série de transtornos para o indivíduo que faz uso desse medicamento, visto que a baixa concentração pode resultar prontamente em uma falha terapêutica que pode comprometer o quadro clínico do paciente (GONDIM et al., 2017).

Os dados desse estudo se assemelham com os achados de Gondim et al (2017) nos quais todas as amostras analisadas não se encontravam dentro do padrão requerido pela Farmacopéia Brasileira. Em contrapartida, Roveri et al (2012) e Oliveira \& Campos (2014) acharam resultados discrepantes em relação ao estudo em questão, ao passo que as amostras analisadas por tais se encontraram dentro do padrão estipulado pela Farmacopéia, excetuando um lote de cápsulas moles $(88,04 \%)$ e de um lote de cápsulas duras manipuladas (78,35\%), do estudo de Roveri et al (2012).

Para evitar que haja esses resultados relacionados a baixo ou alto teor de concentração de princípios ativos, é que os Laboratórios e Indústrias da cadeia Farmacêutica devem se preocupar com a Gestão de Controle de Qualidade no quesito da observância e cumprimento das legislações que regem os princípios de Boas Práticas de Fabricação (BPF) adequando o controle de qualidade mais criterioso e rígido aos resultados, ao passo que esse processo está entrelaçado a saúde pública e diretamente ligado ao usuário desse medicamento que necessita ter sucesso terapêutico (SILVA et al., 2015).

Os elementos de avaliação e gerenciamento dos padrões de qualidade devem ser pautados em cima da infraestrutura adequada, que engloba não apenas instalações físicas, 
mas procedimentos, processos e recursos organizacionais. Além disso, devem ser empregadas ações sistemáticas que assegurem a confiança e integridade de um produto ou serviço e que o mesmo cumpra os requisitos de qualidade (BARRETO, 2017).

A indústria farmacêutica deve ter como base para execução de todos os processos fabris o Manual de Boas Práticas de Fabricação de Medicamentos, pois traz subsídio e medidas necessárias que auxiliam na redução de contaminação-cruzada, contaminação por partículas, troca ou mistura de insumos, riscos que estão intrinsicamente relacionados com os processos de produção de uma indústria farmacêutica (BARRETO, 2017).

\section{Conclusões}

A partir da pesquisa e análise feita, pode-se chegar a conclusão que das amostras de medicamentos utilizadas na pesquisa apenas duas estavam dentro do teor de concentração de princípio ativo ideais e preconizados pela Farmacopeia Brasileira.

De posse desses resultados, pode-se analisar que ainda há muito que se fazer na área de controle de qualidade de grandes indústrias farmacêuticas para garantir que o medicamento chegue para o paciente com todos os requisitos de qualidade assegurados.

Garantir que o medicamento seja produzido sob rigoroso controle e de acordo com as Boas Práticas de Fabricação de Medicamentos repercute diretamente na saúde pública de uma população, ao passo que concentrações superiores ou inferiores de um determinado fármaco podem vir a alterar e prejudicar uma terapia, agravando até o estado do paciente.

\section{Referências}

ANVISA. Farmacopeia Brasileira 5a edição. Brasília: ANVISA, volume 2, 2010. 904p. Disponível em: http://www.anvisa.gov.br/hotsite/cd_farmacopeia/index.htm, Acesso em 10 de setembro de 2018.

BARRETO, L.C.B. Controle de qualidade das análises físico-químicas do paracetamol. 2017. 46f. Trabalho de Conclusão de Curso (Graduação) - Centro Universitário Formiga, Formiga, Minas Gerais, 2017. 
FIGUEIREDO, W.L.M; ALVES, T.C.A. Uso dos anti-inflamatórios não esteroides no controle da do aguda: revisão sistemática. Rev Neurocienc, v.23, n.3, 463-467p, 2015.

GONDIM, A.L.O et al. Análise quantitativa de ibuprofeno em comprimidos de 600 mg disponíveis comercialmente. Revista de Saúde da Faciplac, v.4, n.2, 76-87p., 2017.

OLIVEIRA, V. C. B.; CAMPOS, R. Estudos de equivalência farmacêutica de comprimidos de ibuprofeno. Cadernos da Escola de Saúde- UNIBRASIL, v.11 56-64p., 2014.

ROVERI,F.L et al. Avaliação da aplicação de método espectrofotométrico para determinação do teor de ibuprofeno em diferentes formas farmacêuticas. Rev. Bras. Farm. v.93, n.2, 186-190p., 2012.

SILVA.; G.A et al. Determinação do teor de paracetamol em medicamentos genéricos pela técnica de espectrofotometria UV. REFACER. v.4, n.2, 1-7p., 2015.

SILVA, F.J et al. Controle de qualidade físico-quimico de comprimidos de ibuprofeno 300 mg. Revista Faculdade Montes Belos, v.7, n.1, 151-162p., 2014.

STORPIRTIS, S. et al. Estudo comparativo da cinética de dissolução de ibuprofeno em comprimidos de liberação imediata comercializados no Brasil. Rev. Bras. Cienc. Farm. 35 (2): 281-288, 1999.

Como citar este artigo (Formato ABNT):

SOUZA, Odiney dos Santos; OLIVEIRA, Caio Matheus da Rocha Coqueiro Monteiro. Determinação do teor de Ibuprofeno em Medicamento de Referência, Genérico e Similar, por Espectrofotometria Ultravioleta-Visível, em uma Farmácia Comunitária do Município de Jaguaquara, Bahia. Id on Line Rev.Mult. Psic., 2018, vol.12, n.42, p. 1161-1168. ISSN: 1981-1179.

Recebido: 25/06/2018;

Aceito: 29/10/2018 


\section{ANEXO 01 - PADRÃO DE PUREZA}

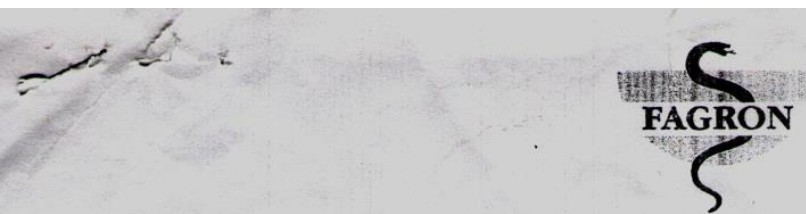

\section{CERTIFICADO DE ANÁLISE}

\begin{tabular}{|c|c|c|c|}
\hline Insumo: & Ibuprofeno & Data de Análise: & $14-10-2016$ \\
\hline Lote Interno: & 16F08-B018-010494 & Lote Fabricante: & $4001 / 1101 / 16 / 0442$ \\
\hline Data de Fabricação: & $01-02-2016$ & Data de Validade: & $01-01-2021$ \\
\hline Origem: & India & Procedência: & India \\
\hline $\begin{array}{l}\text { Condiç̧̄es de } \\
\text { Armazenamento: }\end{array}$ & Temperatura Ambiente & $\begin{array}{l}\text { Ordem de } \\
\text { Fracionamento: }\end{array}$ & 010494 \\
\hline DCB: & 04766 & DCl: & IBUPROFEN \\
\hline CAS: & $15687-27-1$ & Peso Molecular: & 206,2832 \\
\hline Fórmula Molecular: & C13H1802 & & . \\
\hline
\end{tabular}

\begin{tabular}{|c|c|c|c|c|}
\hline Testes & Especificaçōes & Resultados & Unidade & Referências \\
\hline Descrição * & $\begin{array}{l}\text { Pí cristalino, branco ou quase branco, ou cristais } \\
\text { incolores }\end{array}$ & Conforme & & BP - 2016 \\
\hline Identificação * & A: Ponto de fusão 75 a 78 & Conforme & ${ }^{2} \mathrm{C}$ & BP - 2016 \\
\hline \multirow[t]{2}{*}{ Identificaçáo * } & $\begin{array}{l}\text { B: UV Exibe absorçāo máxima } 264 \mathrm{~nm} \text { e } 272 \mathrm{~nm} \text { e } \\
\text { um ombro a } 258 \mathrm{~nm}\end{array}$ & & & \\
\hline & $\begin{array}{l}\text { A razăo de A264/A258 }=1,20-1,30 \text { e } \\
\text { A272/A258=1,00-1,10 }\end{array}$ & Conforme & & BP - 2016 \\
\hline Identificação * & $\begin{array}{l}\text { C: IV-O espectro da amostra está de acordo com o } \\
\text { do padräo. }\end{array}$ & Conforme & & BP - 2016 \\
\hline Identificação * & $\begin{array}{l}\text { D: CCD - A mancha principal obtida no } \\
\text { cromatograma da soluçăo teste é similar em }\end{array}$ & & & \\
\hline 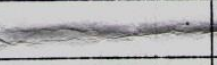 & $\begin{array}{l}\text { posiçäo core tanarhe a mancha principal cbtide ne } \\
\text { cromatiograma corn a soluçăo referência. }\end{array}$ & Contorme & & BP.2016 \\
\hline Solubilidade * & $\begin{array}{l}\text { Praticamente insolúvel em água; facilmente solúvel } \\
\text { em acetona, em metanol e em cloreto de metileno }\end{array}$ & Conforme & & BP - 2016 \\
\hline Solubilidade * & $\begin{array}{l}\text { Dissolve em soluçōes diluídas de hidróxidos } \\
\text { alcaliners e carbonatos }\end{array}$ & Conforme & & BP - 2016 \\
\hline $\begin{array}{l}\text { Perda por } \\
\text { Dessecaçäo : }\end{array}$ & $<=0,5$ (Ambiente/Peso constante à vácuo) & 0,2 & $\%$ & BP - 2016 \\
\hline Cinzas Sulfatadas * & $<=0,1$ & 0,04 & $\%$ & BP - 2016 \\
\hline $\begin{array}{l}\text { Substâncias } \\
\text { Relacionadas(HPLC) * }\end{array}$ & Impureza $A<=0,15$ & Não detectado & $\%$ & BP - 2016 \\
\hline $\begin{array}{l}\text { Substâncias } \\
\text { Relacionadas(HPLC) * }\end{array}$ & Impureza $\mathrm{J}<=0,15$ & Não detectado & $\%$ & BP - 2016 \\
\hline $\begin{array}{l}\text { Substâncias } \\
\text { Relacionadas(HPLC)." }\end{array}$ & Impureza $\mathrm{N}<=0,15$ & Não detectado & $\%$ & BP - 2016 \\
\hline $\begin{array}{l}\text { Substâncias } \\
\text { Relacionadas(HPLC) * }\end{array}$ & Impureza individual $<=0,05$ & Não detectado & $\%$ & BP - 2016 \\
\hline $\begin{array}{l}\text { Substâncias } \\
\text { Relacionadas(HPLC) * }\end{array}$ & Total de Impurezas $<=0,2$ & Não detectado, & $\%$ & BP - 2016 \\
\hline Metais Pesados * & $\Leftrightarrow 10$ & $<10$ & ppm & BP - 2016 \\
\hline Aparência da Soluçăo & A solução $S$ é límpida e incolor & Conforme & & BP - 2016 \\
\hline Rotaçăo optica & $-0,05 \mathrm{a}+0,05$ & 0,00 & - & BP-2016 \\
\hline Impureza $F(C G)$ & $\Leftrightarrow=0,1$ & Năo detectado & $\%$ & BP - 2016 \\
\hline $\begin{array}{l}\text { Solvente } \\
\text { Residual(CG) }\end{array}$ & Acetona $<=100$ & Não detectado & ppm & BP - 2016 \\
\hline
\end{tabular}




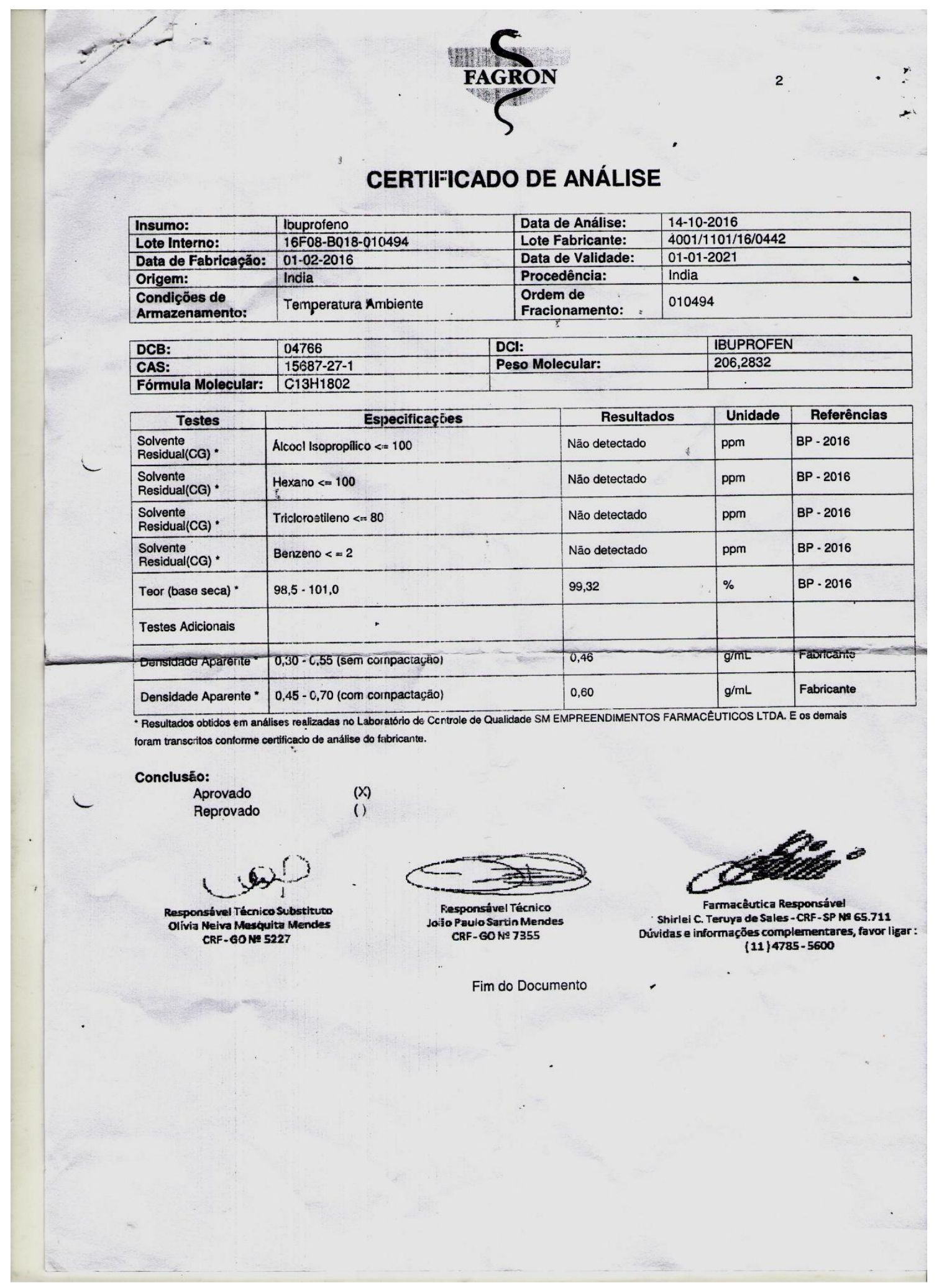

